\title{
Pelvic Floor Relaxation
}

Pelvic floor relaxation-abnormal descent of the bladder (cystocele), uterus and/or vagina (uterine or vaginal vault prolapse), small bowel (enterocele), or rectum (rectocele), is a significant women's health issue affecting primarily parous women $>50$ years of age. Up to $50 \%$ of such women have some degree of genital prolapse. Symptoms are present in $10 \%$ to $20 \%$ of this group and most commonly include pelvic pressure, protrusion of tissue through the pelvic floor and urinary incontinence. In addition to age, risk factors include multiparity, menopause, and obesity. The purpose of this work is to describe a technique for production and review of MR images that can be used to quickly and accurately identify significant pelvic floor defects as an aid to treatment planning (Gold et al., 1999; Fielding et al., 2000).

\section{IMAGING THE PELVIC FLOOR}

In order to achieve an adequate signal-to-noise ratio using the ultra-fast pulse sequences required, MR imaging of the pelvic floor is best done on a high-field strength magnet $(1.0 \mathrm{~T}$ or $1.5 \mathrm{~T})$. The sequences described below are based on the author's experience with a Siemens $1.5 \mathrm{~T}$ vision scanner, but have been applied with success to machines made by other manufacturers. The following 3 sequences comprise the routine protocol for evaluation of pelvic floor descent. When urinary incontinence or uterine prolapse is the primary clinical presentation, no rectal contrast material needs be administered. In the case of global pelvic floor relaxation or suspected rectal prolapse, $60 \mathrm{ml}$ of ultrasound gel should be administered rectally prior to imaging using a catheter-tip syringe. The entire Basic Protocol can be completed in 15 min. There is no need to sedate the patient.

Table A20.3.1 lists the hardware necessary to perform the procedure, along with appropriate parameters. The available gradient strength will depend on the scanner, and the echo times given in other tables below may be varied accordingly (the smaller the gradient strength, the longer the echo time for a particular scan).

NOTE: Be sure that technologists and nurses have immediate access to any emergency equipment that may be relevant to a given study, or that may be needed for a particular patient, such as crash carts or oxygen.

Table A20.3.1 Equipment Parameters for Pelvic Floor Relaxation

\begin{tabular}{ll}
\hline Coil type & $\begin{array}{l}\text { Multi-coil array such as torso or pelvis } \\
\text { phased array coil }\end{array}$ \\
Field strength & 1.0 or $1.5 \mathrm{~T}$ (preferred) \\
Gradient strength & $25 \mathrm{mT} / \mathrm{m}$ (or whatever system permits) \\
Cardiac gating & No \\
Peripheral gating & No \\
Respiratory gating & No \\
Respirator & If required by patient \\
Oxygen & If required by patient \\
Motion cushions & Useful \\
Use of contrast agents & Only for evaluation of rectal prolapse (see \\
& text) \\
\hline
\end{tabular}




\section{Set up patient and equipment}

1. Interview the patient to assess for contraindications such as cardiac pacemakers, implanted mechanical devices, and/or ferromagnetic materials. Also, determine the use of appropriate monitoring equipment.

A screening form (see APPENDIX 1) is signed by each patient or legal guardian prior to bringing the patient into the exam area.

The presence of ferromagnetic materials may be a health hazard to the patient while in the magnetic field and/or adversely affect image quality. If in doubt as to the exact composition of the items, it is best to exclude patients with any metal implants; see Shellock (2001) for discussion of what implants may be safely scanned using magnetic resonance.

Patients may be accompanied into the magnet room by a friend or family member, who can sit in the room during the scan and comfort the patient as needed. This companion must be screened as well to ensure the absence of loose metal objects on the body or clothing.

2. Instruct the patient to change into a gown and remove all personal effects, such as jewelry, hearing aids, glasses, etc., prior to entering the MRI scanning room. Have the patient wash off any mascara and other makeup to avoid local tissue heating and image artifacts. Have the patient void to completion. If the procedure is a research protocol, have the patient sign any necessary consent forms.

3. Explain the procedure to the patient and record relevant clinical history. Ensure that the patient understands what is expected and ask if she has any questions; answer them appropriately.

4. Escort the patient to the MR examination room and review the following items with the patient:

a. Provide earplugs or headphones to the patient to minimize the loud knocking noise that will be produced by the gradient coils but ensure that she will still be able to hear you.

b. Provide the patient with a safety squeeze-bulb and demonstrate how it works; explain to the patient when to use the squeeze-bulb (i.e., if assistance is needed during the exam).

c. Explain to the patient that you will be talking to her between imaging sequences (when the loud knocking noise stops). Additionally, review breath-holding instructions with the patient.

d. Explain to the patient that it is imperative that she remain motionless during the loud knocking noise to ensure good results; also explain that she should not reposition between imaging sequences.

e. Nevertheless, the patient may call out at any time if he or she feels it necessary.

f. Inform the patient with an approximate length of time for the entire examination.

5. Install the bottom portion of the pelvis phased array or torso phased array coil on the table. Instruct the patient to lie down on the table with head toward the scanner, and place a pillow under the patient's knees to reduce discomfort. Secure the top portion of the phased array coil to eliminate it from moving side-to-side during breath-holding imaging sequences.

Usually, straps are provided by the manufacturer that are directly attached to the coil.

6. Using the laser light, center the patient's pelvis in the coil. Include the entire pelvis and uppermost portion of the thighs. Do not sacrifice coil coverage at the level of the upper thighs in order to cover the uppermost portion of the pelvis. 
Table A20.3.2 Primary Clinical Imaging Parameters for Sequence 1 (Pilot Scan)

\begin{tabular}{ll}
\hline Patient position & Supine \\
Scan type & 3-D gradient echo \\
Imaging plane (orientation) & Sagittal, coronal, and transverse \\
Central slice or volume center & Laser light centered on mid pelvis \\
Echo time $\left(T_{\mathrm{E}}\right)$ & As short as possible (e.g., $5 \mathrm{msec})$ \\
Repeat time $\left(T_{\mathrm{R}}\right)$ & As short as possible (e.g., $15 \mathrm{msec})$ \\
Flip angle $(\mathrm{FA})$ & $10^{\circ}$ \\
Fields of view $\left(\mathrm{FOV}_{\mathrm{x}}, \mathrm{FOV}_{\mathrm{y}}\right)$ & $350 \mathrm{~mm}, 350 \mathrm{~mm}$ \\
Resolution $(\Delta x, \Delta y)$ & $1.37 \mathrm{~mm}, 2.73 \mathrm{~mm}$ \\
Number of data points collected $\left(N_{\mathrm{x}}, N_{\mathrm{y}}\right)$ & 256,128 \\
Slice thickness $(\Delta z)$ & $10 \mathrm{~mm}$ \\
Number of slices & 3 per orientation \\
Slice gap & Not applicable \\
Number of acquisitions $\left(N_{\mathrm{acq}}\right)$ & 1 \\
Swap read and phase encoding & Standard default \\
Scan time & $\sim 15 \mathrm{sec}$ \\
\hline
\end{tabular}

7. Advance the patient table to isocenter.

\section{Sequence 1: Rapid three-plane positioning pilot}

8. Instruct the patient to take in a deep breath and exhale, take in another deep breath, and then hold it. Initiate the scan as in step 9.

9. Run the system's pilot (or scout) scan to ensure correct location of the pelvis in three directions using the imaging sequence given in Table A20.3.2.

Please refer to manufacturer's specifications for standard default setting and swap read and phase encoding directions as necessary.

\section{Sequence 2: Sagittal $T_{2}$-weighted midline pelvis images at rest and at strain}

In general, a woman can only hold maximal downward pelvic strain for $7 \mathrm{sec}$. For this reason, ultra-fast pulse sequences must be employed. Common acronyms are single-shot fast-spin echo (SS-FSE; GE) and half acquisition single-shot turbo-spin echo (HASTE; Siemens). These pulse sequences perform phase encoding of all data simultaneously and sample only half of $k$-space.

10. Use the transverse and sagittal images from sequence 1 to choose a midline image that shows the symphysis, bladder neck, cervix, and coccyx.

In order to determine which of the three main compartments of the pelvic floor descend abnormally, it is imperative that all be encompassed on a single slice.

11. Instruct the patient to take in a deep breath and exhale, take in another deep breath, and then hold it.

12. Run sequence 2 according to Table A20.3.3 while the patient's pelvic floor is at rest.

13. Instruct the patient to take in a deep breath and exhale, take in another deep breath, and hold it.

14. Coach the patient verbally to strain her pelvis maximally downward and re-run sequence 2 according to Table A20.3.3.

If an adequate strain is not obtained, repeat steps 13 and 14 with further coaching. 
Table A20.3.3 Primary Clinical Imaging Parameters for Sequence 2 (SSFSE or HASTE)

$\begin{array}{ll}\text { Patient position } & \text { Supine } \\ \text { Scan type } & \text { Single-shot fast-spin echo } \\ \text { Imaging plane (orientation) } & \text { Sagittal } \\ \text { Central slice or volume center } & \text { Centered on bladder base } \\ \text { Echo time }\left(T_{\mathrm{E}}\right) & 90 \mathrm{msec} \\ \text { Repeat time }\left(T_{\mathrm{R}}\right) & 4.4 \mathrm{msec} \text { (the true } T_{\mathrm{R}} \text { is infinite, } 4.4 \\ & \mathrm{msec} \text { represents the echo spacing) } \\ \text { Flip angle (FA) } & 180^{\circ a} \\ \text { Fields of view }\left(\mathrm{FOV}_{\mathrm{x}}, \mathrm{FOV} \mathrm{y}\right) & 350 \mathrm{~mm}, 350 \mathrm{~mm} \\ \text { Resolution }(\Delta x, \Delta y) & 1.37 \mathrm{~mm}, 2.06 \mathrm{~mm} \\ \text { Number of data points collected }\left(N_{\mathrm{x}}, N_{\mathrm{y}}\right) & 256,170 \\ \text { Display matrix }\left(D_{\mathrm{x}}, D_{\mathrm{y}}\right) & 512,512 \\ \text { Slice thickness }(\Delta z) & 10 \mathrm{~mm} \\ \text { Number of slices } & 1 \\ \text { Slice gap } & \text { Not applicable } \\ \text { Number of acquisitions }\left(N_{\mathrm{acq}}\right) & 0.5 \text { (half Fourier) } \\ \text { Read direction } & \text { Anterior-posterior } \\ \text { Slice locations } & \text { Midline pelvis } \\ \text { Saturation pulses } & \text { No } \\ \text { Scan time } & 1 \text { sec (the total scan time is } 2 \mathrm{sec}, 1 \text { at } \\ & \text { rest and 1 at strain, see text) }\end{array}$

$\overline{{ }^{a}}$ The system displays the flip angle of the refocusing pulse. The flip angle of the first pulse of this sequence is $90^{\circ}$.

Table A20.3.4 Primary Clinical Imaging Parameters for Sequence 3 (FSE or TSE)

Patient position

Scan type

Imagine plane (orientation)

Central slice or volume center

Echo time $\left(T_{\mathrm{E}}\right)$

Echo train length (ETL)

Repeat time $\left(T_{\mathrm{R}}\right)$

Flip angle (FA)

Fields of view $\left(\mathrm{FOV}_{\mathrm{x}}, \mathrm{FOV}_{\mathrm{y}}\right)$

Resolution $(\Delta x, \Delta y)$

Number of data points collected $\left(N_{\mathrm{x}}, N_{\mathrm{y}}\right)$

Display matrix $\left(D_{\mathrm{x}}, D_{\mathrm{y}}\right)$

Slice thickness $(\Delta z)$

Number of slices

Slab thickness

Slice gap

Number of acquisitions ( $\left.N_{\text {acq }}\right)$

Read direction

Slice locations

Saturation pulses

Fat supression

Slice series

Scan time
Supine

FSE

Transverse

Centered on midpoint of pubococcygeal line

$132 \mathrm{msec}$

8

$5000 \mathrm{msec}$

$180^{\circ} a$

$240 \mathrm{~mm}, 180 \mathrm{~mm}$

$0.67 \mathrm{~mm}, 0.47 \mathrm{~mm}$

360,384

512,512

$3 \mathrm{~mm}$

19

$57 \mathrm{~mm}$

$0 \mathrm{~mm}$

2

Anterior-posterior

Pelvic floor

None

No

Interleaved

$\sim 7 \mathrm{~min}$

${ }^{a}$ The system displays the flip angle of the refocusing pulse. The flip angle of the first pulse of this sequence is $90^{\circ}$. 
Sequence 3: Transverse fast spin echo (FSE or TSE, turbo spin echo) images of the pelvic floor

15. After completion of sequence 2 , review the resting image and identify the pelvic floor.

The pelvic floor usually extends from the last joint of the coccyx to the inferiormost aspect of the symphysis.

16. Use console calipers, or visually estimate a line drawn between the aforementioned two points (the pubococcygeal line).

17. Instruct the patient that she may breathe normally for the next series.

18. Use sequence 3 to identify signal changes and tears within the supporting muscles of the pelvic floor. Run the sequence according to Table A20.3.4.

\section{COMMENTARY}

\section{Background Information}

For the majority of patients with mild to moderate stress incontinence and pelvic floor relaxation, the combination of physical examination findings and urodynamic pressure readings is diagnostic and no further imaging is required. For those patients with severe urinary or fecal incontinence thought to be multifactorial, multiple compartment involvement, or have failed prior surgery, imaging can be extremely valuable.

There are several techniques that can be used to evaluate the pelvic organs. These include voiding cystourethrography (with or without concurrent video urodynamic tracings), ultrasound of the bladder neck and anal sphincter, colpocystodefecography, and magnetic resonance imaging.

\section{Voiding cystourethrography}

Voiding cystourethrography (VCUG) is often performed during an incontinence workup to exclude anatomic abnormalities such as duplicated collecting systems and ureters, bladder and urethral diverticula, and vesicoureteral reflux. Even when these findings are not present, examination of the bladder can provide useful information. Trabeculation is a sign of urge incontinence, or detrusor overactivity (Fielding, 2002). The hallmark of this disease is the sudden onset and imminent need to void due to a bladder contraction. This is the most common type of incontinence in the elderly, affecting up to $30 \%$ of those living at home and $50 \%$ in long-term care institutions. Identification of an unsuspected neurogenic bladder is another significant finding. These patients often have a history of spinal injury. The bladder remains contracted and severely trabeculated during the entirety of the exam and may have a markedly tapered dome. While VCUG is rarely required to diagnose stress incontinence, it can be useful in severe cases when the bladder extends so far inferiorly that bladder neck kinks, masking symptoms.

\section{Ultrasound}

Ultrasound of the bladder neck or perineum is an alternative way to diagnose stress incontinence. Again, it is usually only of clinical use for assessment of bladder neck mobility. Trans rectal ultrasound (TRUS) has been shown to be reliable in the identification of rectal sphincter tears and atrophy (Gold et al., 1999). It is often used prior to surgery to gauge the size and depth of tear and identify adjacent hypoechoic scar tissue. A small probe providing circumferential images is placed in the rectal canal. Images are obtained at 5-mm intervals along the length of the sphincter, $\sim 2.5$ $\mathrm{cm}$. The normal internal sphincter is a circumferential black band of uniform thickness. The external sphincter is harder to see because it is echogenic. Tears are defined as areas of discontinuity. Large tears respond poorly even to aggressive surgical intervention.

\section{Defecography}

Colpocystodefecography is a method of observing all three compartments of the pelvic floor during rest, upward contraction, and Valsalva. Because it is done in the sitting position, it most closely mimics the physiologic state (Kelvin, 1998). In most institutions, it is used primarily to identify posterior compartment abnormalities, therefore the bladder is not opacified. One hour prior to the study, the patient is given a barium meal to coat the small bowel loops. Barium paste is placed into the rectum, usually with the aid of a caulking gun, and the patient places a tampon soaked in contrast material in the vagina. In the upright position, multiple spot fluoroscopic images are
Female Pelvis

A20.3.5

Supplement 8 

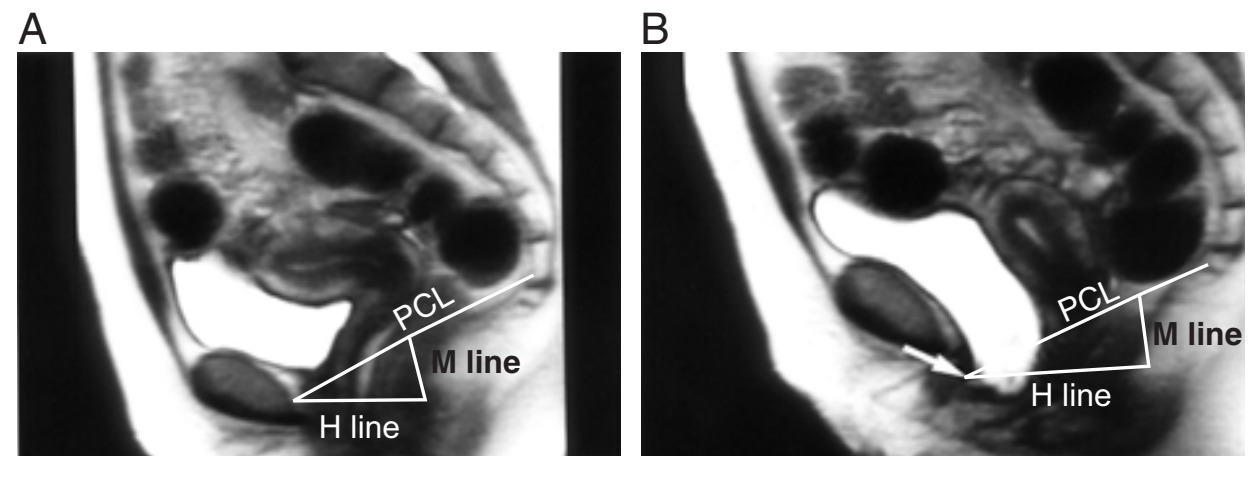

Figure A20.3.1 Sagittal MR images of a 55-year-old woman with stress incontinence and incomplete bladder emptying. Sagittal $T_{2}$-weighted image at rest $(\mathbf{A})$ shows pelvic organs and floor to have normal appearance. On strain (B), there is development of a cystocele and rotation of the urethra into the horizontal plane (arrow) indicating damage to the internal sphincter. Reproduced from Fielding (2002) with permission from Radiographics.

obtained during pelvic floor maneuvers, concluding with defecation. With a pelvic floor contraction, a sharp anorectal angle is an indicator of good muscular support, however, specific measurements have not been found useful. Retained barium within a portion of the anterior rectum that bulges $>2 \mathrm{~cm}$ into the rectovaginal space is defined as an anterior rectocele. $\mathrm{Pa}$ tients will often be able to manually empty the rectocele. Descent of small bowel loops into the rectovaginal space is defined as an enterocele and indicates a tear in the rectovaginal fascia. Many gastroenterologists believe that enterocele gives a patient the feeling of incomplete defecation despite the presence of an empty rectum leading to persistent and ineffectual straining. Intusussception usually involves only the rectum or rectosigmoid and resolves with cessation of Valsalva.

\section{Magnetic resonance imaging}

During the past 10 years, magnetic resonance imaging (MRI) has emerged as a competitor to other imaging modalities for evaluation of the female pelvic floor. The main advantages of MRI are the ability to evaluate the three compartments of the pelvic floor simultaneously during rest and strain and the direct visualization of supporting structures (Fielding et al., 1998; Unterweger et al., 2001). Disadvantages include the requirement that the exam be performed in the supine or left lateral decubitus position, although one group working with an open configuration magnet reported no significant difference between upright and supine findings (Bertschinger et al., 2002).

\section{Critical Parameters and Troubleshooting}

The two most common reasons for poor quality MR images of the pelvic floor are (1) a patient's inability to perform adequate pelvic strain, and (2) incorrect patient positioning for obtaining the critical midline sagittal images.

Before beginning the exam and after the patient is situated in the magnet, it is helpful to practice pelvic strain. Instruct the patient to take a slow deep breath in and then bear down as if she is having a bowel movement. Ask her to keep her buttocks and thighs on the table while she strains. During the exam, if the operator suspects that an adequate strain is not obtained, simply repeat the sagittal scan following further coaching.

Reinforce with the technologists the necessity of absolute midline positioning. If the scout does not demonstrate all of the midline pelvic structures, the patient must be repositioned so that she rests absolutely flat on the table. If the scout is difficult to read, the author often finds it helpful to expand the first sagittal HASTE series (sequence 2) to 3 or 4 slices in order to find the best midline image.

\section{Anticipated Results}

On sagittal images, the pubococcygeal (PCL) line should be drawn between the last joint of the coccyx and the inferiormost aspect of the symphysis. Urologists and gynecologists use this line as an indicator of the pelvic floor. In an early work, Yang et al. (1991) used gradient echo images to define maximal normal descent of the bladder base $(1.0 \mathrm{~cm}$ below), 
vagina (1.0 cm above), and rectum $(2.5 \mathrm{~cm}$ below) with respect to the pubococcygeal line. In practical terms, descent of the bladder or vagina $>1 \mathrm{~cm}$ below the PCL indicates some degree of laxity while descent $>2 \mathrm{~cm}$ in asymptomatic patient often requires surgical therapy (Fig. A20.3.1). Rectal abnormalities such as anterior rectocele and intussusception as well as enterocele are identified in the same fashion as with defecography. There are other important findings on sagittal images. The levator plate should remain parallel to the pubococcygeal line at all times. Caudal angulation of the levator plate $>10^{\circ}$ indicates loss of pelvic floor support (Ozasa et al., 1992; Hoyte et al., 2001).

Measurements of the $\mathrm{H}$ and $\mathrm{M}$ lines are useful ways to quantify loss of pelvic floor support (Comiter et al., 1999). The H line is drawn from the inferior aspect of the symphysis pubis to the posterior wall of the rectum and measures the anterior-posterior dimension of the pelvic hiatus. The $\mathrm{M}$ line is drawn as a perpendicular from the PCL to the posteriormost aspect of the $\mathrm{H}$ line. It measures the height of the hiatus. In healthy women, the $\mathrm{H}$ line should not exceed $5 \mathrm{~cm}$ and the $\mathrm{M}$ line should not exceed $2 \mathrm{~cm}$. Values greater than these indicate loss of pelvic floor support.

Transverse images should be reviewed for muscle integrity and signal intensity and for the vaginal shape and location. The puborectalis should extend from the parasymphysial insertion posterior to the rectum. It should be of similar width along its entire course without evidence of gaps or fraying. The width of the levator hiatus at the level of the symphysis rarely exceeds $4.5 \mathrm{~cm}$ in healthy volunteers, however, there is some overlap with incontinent patients. The vagina should normally be of $\mathrm{H}$ or butterfly shape and centered in the pelvis (Klutke et al., 1990).

\section{Literature Cited}

Bertschinger, K.M., Hetzer, F.H., Roos, J.E., Treiber, K., Marincek, B., and Hilfiker, P.R. 2002. Dynamic MR imaging of the pelvic floor performed with patient sitting in an open-magnet unit versus with patient supine in a closed-magnet unit. Radiology 223:501-508.

Comiter, C.V., Vasavada, S.P., Barbaric, Z.L., Gousse, A.E., and Raz, S. 1999. Grading pelvic prolapse and pelvic floor relaxation using dynamic magnetic resonance imaging. Urology $3: 454-457$.
Fielding, J.R. 2002. Practical MR imaging of female pelvic floor weakness. Radiographics 22:295304.

Fielding, J.R., Griffiths, D.J., Versi, E., Mulkern, R.V., Lee, M.L., and Jolesz, F.A. 1998. MR imaging of pelvic floor continence mechanisms in the supine and sitting positions. A.J.R. 171:1607-1610.

Fielding, J.R., Lee, J.H., Dubeau, C.E., Zou, K.H., and Resnick, N.M. 2000. Voiding cystourethrography findings in elderly women with urge incontinence. J. Urol. 163:1216-1218.

Gold, D.G., Halligan, S., Kmiot, W., and Bartram, C.I. 1999. Anal endosonography: Inter- and intra-observer agreement. Br. J. Surg. 86:371-375.

Hoyte, L., Schierlitz, L., Zou, K., Flesh, G., and Fielding, J.R. 2001. Two- and 3-dimensional MRI comparison of levator ani structure, volume, and integrity in women with stress incontinence and prolapse. Am. J. Obstet. Gynecol. 185:11-19.

Kelvin, F.M., Maglinte, D.D., Hornback, J.A., and Benson, J.T. 1998. Pelvic prolapse: Assessment with evacuation proctography (defecography). Radiology 184:547-551.

Klutke, C., Golomb, J., Barbaric, Z., and Raz, S. 1990. The anatomy of stress incontinence: Magnetic resonance imaging of the female bladder neck and urethra. J. Urol. 143:563-566.

Ozasa, H., Mori, T., and Togashi, K. 1992. Study of uterine prolapse by magnetic resonance imaging: Topographical changes involving the levator ani muscle and the vagina. Gynecol. Obstet. Invest. $24: 43-48$.

Shellock, F.G. 2001. Pocket Guide to MR Procedures and Metallic Objects. Lippincott-Raven, Philadelphia.

Unterweger, M., Marincek, B., Gottstein-Aalame, N., Debatin, J.F., Seifert, B., Ochsenbein-Imhof, N., Perucchini, D., Kubik-Huch, R.A. 2001. Ultrafast MR imaging of the pelvic floor. A.J.R.176:959-963.

Yang, A., Mostwin, J.L., Rosenshein, N.B., and Zerhouni, E.A. 1991. Pelvic floor descent in women: Dynamic evaluation with fast MR imaging and cinematic display. Radiology 179:2533.

\section{Internet Resource}

http://www.mrisafety.com

This is the definitive site for MR safety including medical devices. It is free to all users following registration.

Contributed by Julia R. Fielding

University of North Carolina at Chapel Hill Chapel Hill, North Carolina
Female Pelvis

A20.3.7

Supplement 8 